\title{
Numerical Investigation Into a Surface Plasmon Resonance Sensor Based on Optical Fiber Microring
}

\author{
Chunliu ZHAO*, Yanru WANG, Dongning WANG, and Zhewen DING \\ Institute of Optoelectronic Technology, China Jiliang University, Hangzhou, 310018, China \\ *Corresponding author: Chunliu ZHAO \\ E-mail: clzhao@cjlu.edu.cn
}

\begin{abstract}
A reflective surface plasmon resonance (SPR) sensor based on optical fiber microring is proposed. In such a sensor, plasmons on the outer surface of the metallized channels containing analyte can be excited by a fundamental mode of a thin-core fiber (TCF). The refractive index (RI) sensing can be achieved as the surface plasmons are sensitive to changes in the refrective index of the analyte. Numerical simulation results show that the resonance spectrum shifts toward the shorter wavelength gradually when the analyte refractive index increases from 1.0 to 1.33 , whereas it shifts toward the longer wavelength gradually when the analyte refractive index increases from 1.33 to 1.43 , and there is a turning point at the refractive index value of 1.33 . The highest sensitivity achieved is up to $2.30 \times 10^{3} \mathrm{~nm} / \mathrm{RIU}$ near the refractive index value of 1.0 . Such a compact sensor has potential for gaseous substance monitoring.
\end{abstract}

Keywords: Fiber optics; surface plasmons; sensors; microstructure

Citation: Chunliu ZHAO, Yanru WANG, Dongning WANG, and Zhewen DING, "Numerical Investigation Into a Surface Plasmon Resonance Sensor Based on Optical Fiber Microring," Photonic Sensors, 2017, 7(2): 105-112.

\section{Introduction}

Surface plasmons propagating at the metal-dielectric interface are extremely sensitive to changes in the refractive index of the dielectric medium. This feature constitutes the core of many surface plasmon resonance (SPR) sensors [1]. Typically, these sensors are implemented in the Kretschmann configuration [2] to direct $p$-polarized light through a glass prism and reflect it from a thin metal $(\mathrm{Au}$ and $\mathrm{Ag})$ film deposited on the prism facet. However, this method remains a gold standard for commercial SPR systems, which is bulky, expensive, and not suitable for some applications such as remote sensing. To overcome these limitations, using optical fiber instead of a prism has attracted a lot of research attention due to its advantages of miniaturization, label-free sensing, remote sensing capabilities, and real-time monitoring. The SPR optical fiber sensors have board applications in biology, environment, chemistry, medicine, etc. [3-8]. Over the past decade, many fiber-based SPR sensors have been reported [9-14], including sensors based on side-polished fiber, D-type fiber, tapered fiber, and fiber Bragg gratings. These sensors are based on Kretschmann [2] configuration, to mentallize fiber surfaces and enable evanescent coupling with a plasmon, and one has to first strip the fiber jacket and then etch or taper fiber cladding almost to the core. This laborious procedure compromises fiber integrity, making the resultant sensor be prone to mechanical failures. In recent years, with the rapid development of the micromachining technology, many microstructures

Received: 15 June 2016 / Revised: 12 November 2016

(C) The Author(s) 2017. This article is published with open access at Springerlink.com

DOI: $10.1007 / \mathrm{s} 13320-017-0359-7$

Article type: Regular 
on optical fiber have been fabricated and used as microfluidic channels for the analyte measurement. SPR sensors based on microstructured optical fibers (MOFs) have attracted immense research interest worldwide [15-20]. Deposition of metal layers inside of the MOFs can be performed either with the high-pressure chemical vapor deposition technique [21] or with electroless plating techniques used for the fabrication of metallized hollow waveguides and microstructures [22, 23]. Microfluidics on microstructured fibers is enabled by passing analyte though the fiber porous cladding, thus, partially solving the packaging problem.

In this paper, we present a reflective SPR sensor based on an MOF by introducing a microring as the microfluidic channel into the cladding of the thin-core fiber (TCF). A gold layer is deposited on the outer surface of the microring to form the Otto configuration [2]. As far as we know, no experimental observations and theoretical analysis on optical fiber SPR sensors based on the Otto configuration have been reported. The excitation of plasmon resonances in the proposed sensor is investigated numerically using the finite element method (FEM). Numerical simulation results show that such a compact structure can excite SPR effectively. The resonance spectrum shifts toward the shorter wavelength gradually when the refractive index (RI) of the analyte increases from 1.0 to 1.33 and shifts toward the longer wavelength gradually when the RI of the analyte increases from 1.33 to 1.43 , and there is a turning point at the RI value of 1.33 , which is a phenomenon not observed in other structures. The highest sensitivity achieved is $2.30 \times 10^{3} \mathrm{~nm} / \mathrm{RIU}$ near the RI value of 1.0. Such a fiber microring SPR sensor has a large measurement range, compact size, and a durable structure which can be used in gaseous material detections.

\section{Geometry and theory}

Figure 1 shows the cross-sections of the proposed SPR sensor based on optical fiber microring. This kind of structure design is based on
$[24,25]$, in which an air ring is introduced as the microfluidic channel into the cladding of the TCF. A gold layer with thickness $50 \mathrm{~nm}$ is desposited on the outer surface of the microring. The reflective type sensor is formed by a layer of gold film with thickness $50 \mathrm{~nm}$ coated on the optical fiber end. The sensing head is composed of a core layer, a residual cladding layer, an air layer, a metal layer, and a cladding layer, going from inside to outside. The thickness is $50 \mathrm{~nm}$ for both the residual cladding and the air ring. The air ring can be used as a microfluidic channel, in which the tested samples with different RIs can be injected directly. In the simulation, the diameter and the RI of the fiber core are selected as $7 \mu \mathrm{m}$ and 1.46 , respectively, and the cladding $\mathrm{RI}$ is 1.45 .

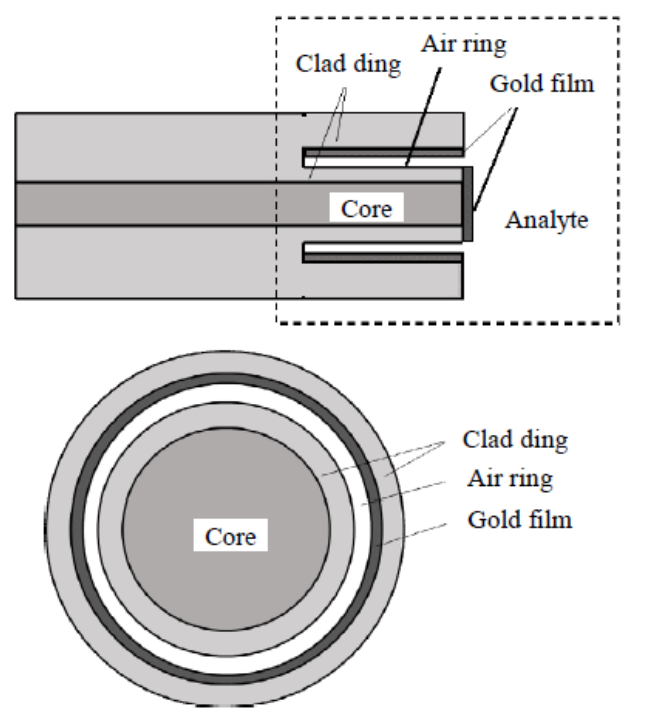

Fig. 1 Cross-sectional structure of the SPR sensor head based on optic fiber microring.

When light propagates in the fiber, due to the reduction of the fiber cladding, the fundamental mode spreads out of the residual cladding region and forms a dynamic evanescent field. In the SPR setup based on the Otto configuration, the surface plasma wave (SPW) is supported by the tail of the evanescent wave at the metal-microring (analyte) interface. Owing to the strong absorption of the metal film, there are lots of plasmon charges at the interface, and the SPW propagates along the metal-microring (analyte) boundary. Furthermore, 
the SPR can be excited when the light wave vector matches the SPW vector at the plane of $z=0$ [2], leading to local field reinforcement at the interface between the gold film and the sensing sample. When light propagates to the fiber end, the light is reflected due to the presence of the gold layer coated on the fiber end. The reflected light passes through the mental-microring interface again to reinforce the SPR effect. The SPR effect in the surface of the metal film is easy to be influenced by the surrounding medium, and the position of the SPR spectrum is changed with the RI of the analyte injected into the microring. Thus, by detecting the wavelength shift, the RI of the analyte can be measured.

\section{Numerical results and discussion}

Commercial software COMSOL Multiphysics with perfectly matched layer boundaries based on FEM was used to analyze the proposed sensor. Figure 2 illustrates the intensity of the electric field in the cross section of the optical fiber microring structure using the study "mode analyses" from COMSOL Multiphysics. The wavelength of the light is $460 \mathrm{~nm}$. As shown in Fig. 2(a), the electric field intensity at the interface between the gold film and the microring is obviously reinforced when the gold film is deposited and SPR is excited (the thickness of $\mathrm{Au}$ film $=50 \mathrm{~nm}$, analyte RI $n_{a}=1.33$ ), while in Fig. 2(b), no SPR is excited as the absence of the gold film. It means that the proposed sensing structure is advisable to excite the SPR effect.

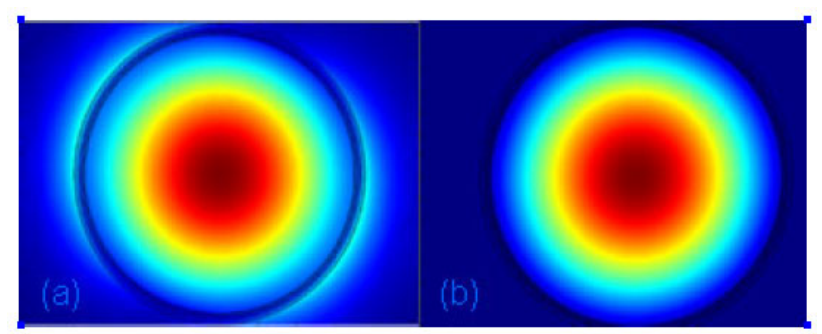

(a)

(b)

Fig. 2 Electric field distribution in the cross section of the fiber microring at the wavelength $\lambda=460 \mathrm{~nm}$ : (a) when the SPR is excited with the gold film thickness of $50 \mathrm{~nm}$ and analyte RI is 1.33 and (b) no SPR is excited in the absence of the gold film.
The performance of the sensor depends on its structural parameters. According to the SPR theory based on the Kretschmann configuration, due to the radiation damping, if the thickness of the metal is too small, the SPW at the metal surface will be strongly damped. While the thickness of the metal is too large, the SPW can no more be efficiently excited because of the strong absorption of the metal [2]. Thus, the thickness of the $\mathrm{Au}$ film has significant effect on the surface plasmon wave excitation. To observe the effect of Au film thickness on the sensor performance, the Au film thickness is varied from $40 \mathrm{~nm}$ to $70 \mathrm{~nm}$ while other parameters remain as constant (residual cladding thickness= $50 \mathrm{~nm}$, air ring thickness $=50 \mathrm{~nm}$, and $n_{a}=1.0$ ) in the simulation. When using COMSOL Multiphysics software, the electric field intensity $(E)$ at different wavelengths can be obtained. The corresponding intensity $(I)$ is obtained by the following equation

$$
I(w)=\frac{1}{2} c \varepsilon_{0} E^{2}
$$

where $c$ is the speed of light; $\varepsilon_{0}$ is the permittivity of vacuum; $E$ is the electric field intensity. And then the intensity in $\mathrm{dBm}$ is transformed form the following formula

$$
I(\mathrm{dBm})=10 \cdot \lg \left[\frac{I(w)}{1 m w}\right] .
$$

All calculations and SPR spectra are performed by using Matlab. The SPR spectra corresponding to different $\mathrm{Au}$ film thicknesses are shown in Fig. 3, where it can be observed that the resonance wavelength shifts toward longer wavelength with an increase in thickness of the $\mathrm{Au}$ film. This indicates that due to an increase in $\mathrm{Au}$ film thickness, light penetration through the cladding decreases. The gold film thickness of $50 \mathrm{~nm}$ is selected for the RI sensor simulation.

Besides the $\mathrm{Au}$ film thickness, effects of the microring thickness on the SPR spectrum has also been studied (residual cladding thickness $=50 \mathrm{~nm}, \mathrm{Au}$ film thickness $=50 \mathrm{~nm}$, and $n_{a}=1.0$ ), and the relevant graphs obtained are shown in Fig. 4, where 
it can be seen that the resonance peak shifts toward longer wavelength when the microring thickness increases from $20 \mathrm{~nm}$ to $60 \mathrm{~nm}$. The air ring thickness of $50 \mathrm{~nm}$ is selected for the RI sensor simulation.

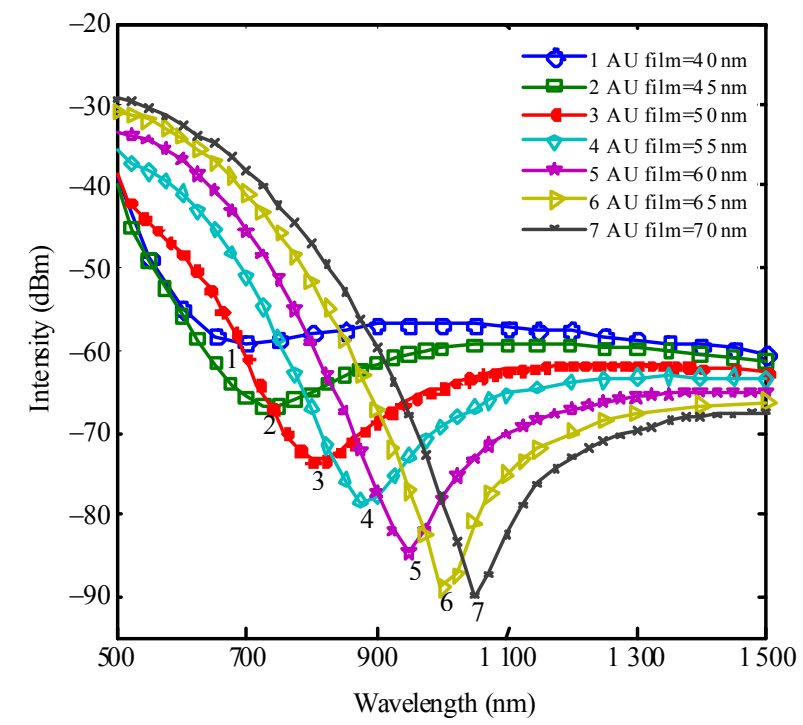

Fig. 3 SPR spectra with different thicknesses of gold film (residual cladding thickness $=50 \mathrm{~nm}$, air ring thickness $=50 \mathrm{~nm}$, and $n_{a}=1.0$ ).

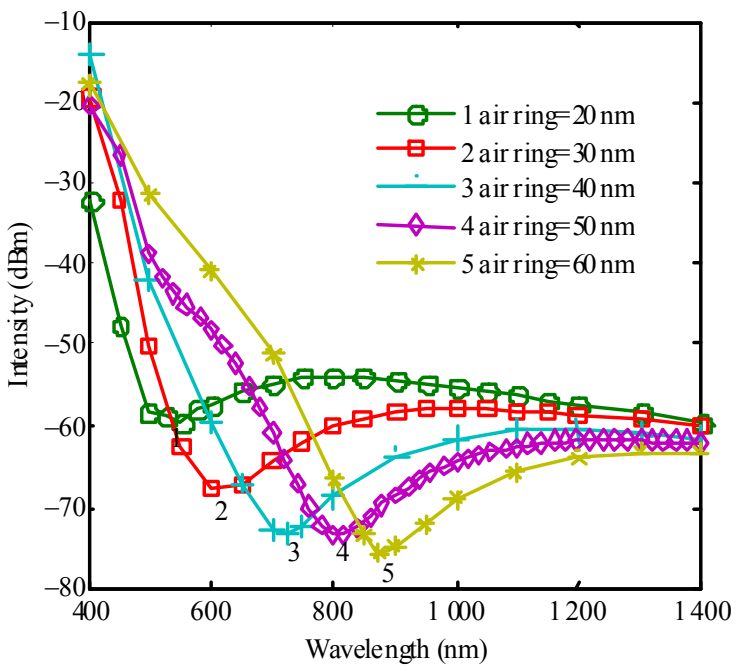

Fig. 4 SPR spectra with different thicknesses of air ring (residual cladding $=50 \mathrm{~nm}, \mathrm{Au}$ film $=50 \mathrm{~nm}$, and $n_{a}=1.0$ ).

In addition, the influence of the thickness of the remaining cladding near the fiber core $(\mathrm{Au}$ film thickness $=50 \mathrm{~nm}$, air ring thickness $=50 \mathrm{~nm}$, and $n_{a}=1.0$ ) on the SPR resonance wavelength is demonstrated in Fig. 5. It can be seen from Fig. 5 that the thickness of the remaining cladding near the fiber core has little effect on the SPR spectrum.

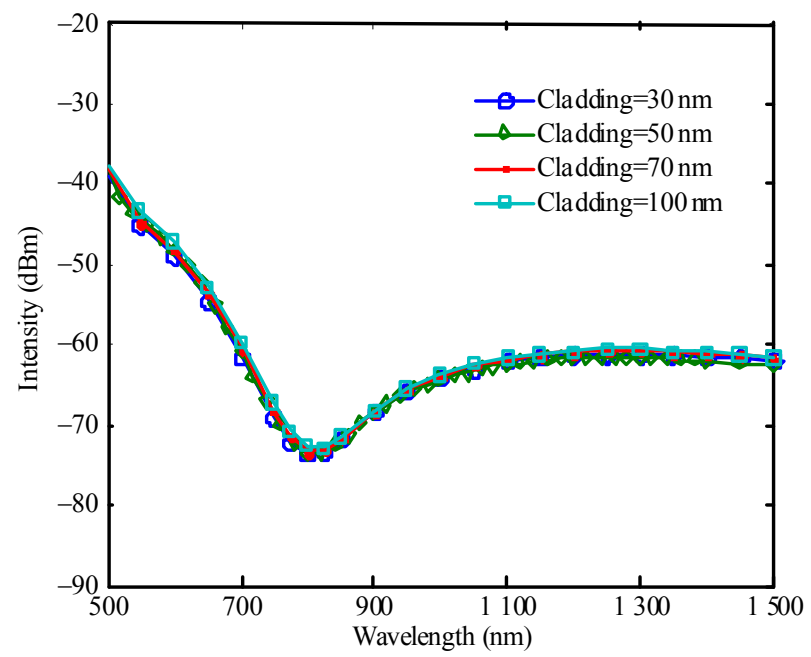

Fig. 5 SPR spectra with different thicknesses of the remaining cladding near the fiber core $(\mathrm{Au}$ film thickness $=50$ $\mathrm{nm}$, air ring thickness $=50 \mathrm{~nm}$, and $n_{a}=1.0$ ).

The results of the resonant wavelength shift obtained by varying the analyte RI from 1.0 to 1.43 are revealed in Fig. 6, where the thicknesses of the residual cladding, gold film, and the microring are all equal to $50 \mathrm{~nm}$. It can be observed from Fig. 6 that there is a turning point in the change of resonance wavelength with the RI at the RI value of 1.33. In Fig. 6(a), the resonance spectrum shifts toward the shorter wavelength gradually when the analyte RI increases from 1.0 to 1.33 . The relationship between the resonant wavelength and the analyte RI in the range of $1.0-1.3$ is shown in Fig. 6(b). The corresponding regression equation is

$$
y=3.179 \times 10^{3} x^{2}-8.486 \times 10^{3} x+6.117 \times 10^{3}
$$

where $y$ is the resonant wavelength in $\mathrm{nm}$, and $x$ is the analyte RI $\left(n_{a}\right)$. The resonant wavelength shifts from $815 \mathrm{~nm}$ to $700 \mathrm{~nm}$ when the $n_{a}$ varies from 1.0 to 1.05 . The positive RI sensitivity $(S)$ obtained is $2.30 \times 10^{3} \mathrm{~nm} / \mathrm{RIU}$, determined by the following equation

$$
S=\left|\frac{d \lambda_{R}}{d n_{a}}\right|
$$

where $d \lambda_{R}$ is the resonant peak shift, and $d n_{a}$ is the analyte RI variation. The proposed sensor shows the sensitivity of $2300 \mathrm{~nm} / \mathrm{RIU}, 1600 \mathrm{~nm} / \mathrm{RIU}$, $1200 \mathrm{~nm} / \mathrm{RIU}$, and $600 \mathrm{~nm} / \mathrm{RIU}$ for analyte RI variation ranges of 1.0 to $1.05,1.05$ to $1.1,1.1$ to 1.2 , and 1.2 to 1.33 , respectively. 


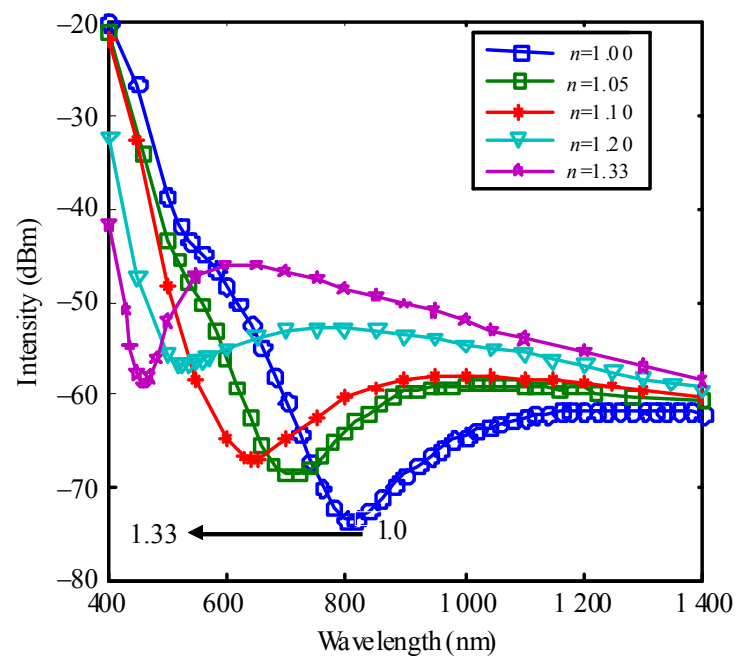

(a)

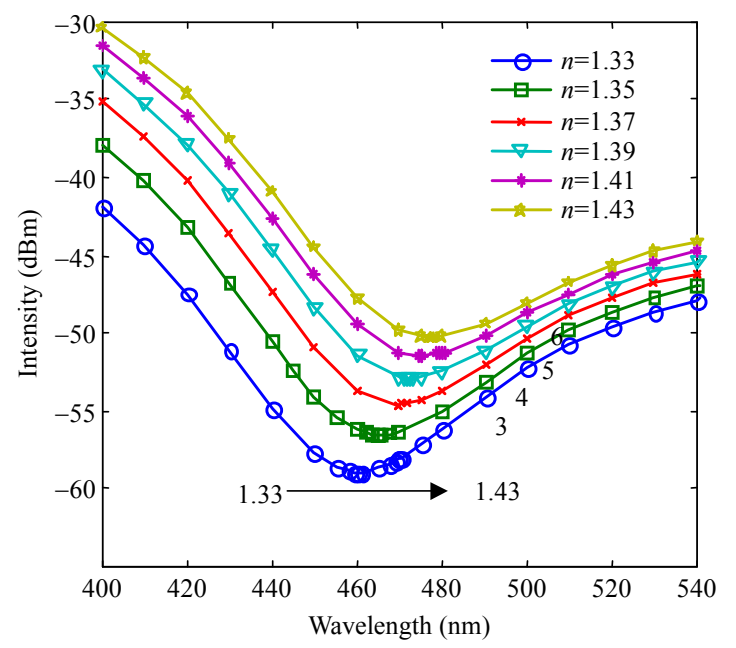

(c)

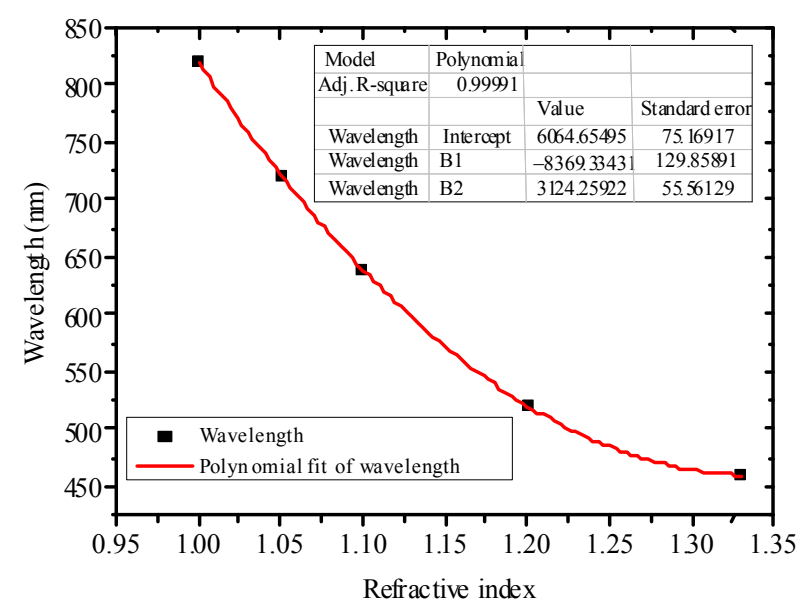

(b)

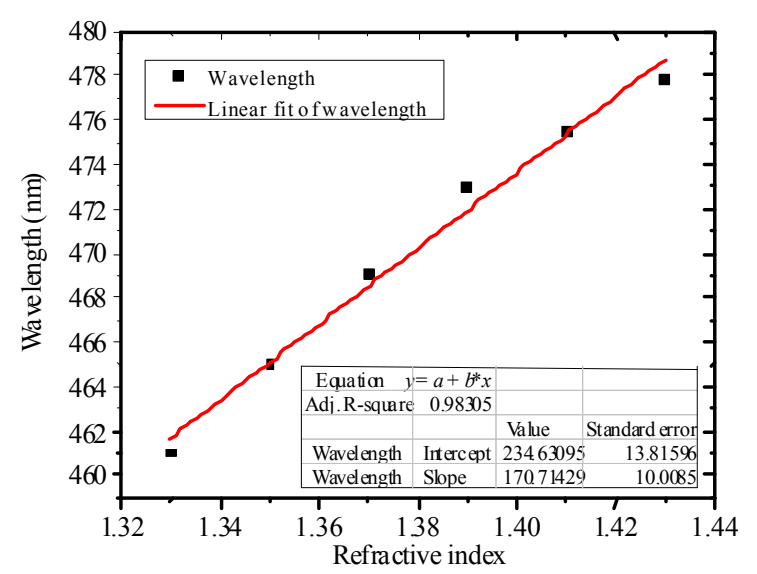

(d)

Fig. 6 SPR spectra with analyte RI variation: (a) from 1.0 to 1.33 , (c) from 1.33 to 1.43 , and (b) and (d) the relationship between resonant wavelength and analyte RI.

It can be seen from Fig. 6(c) that the resonance spectrum shifts toward the longer wavelength gradually when the analyte RI increases from 1.33 to 1.43. Figure $6(\mathrm{~d})$ shows the linear line fitting of the resonant wavelength with respect to the analyte RI, and the regression equation is

$$
y=170.71 x+234.63,1.33 \leq x \leq 1.43
$$

where $y$ is the resonant wavelength of the analyte in $\mathrm{nm}$, and $x$ is the analyte RI. The average sensitivity is $170 \mathrm{~nm} / \mathrm{RIU}$, and $R^{2}$ is 0.9830 , indicating a good fitting of the sensor response.

Theoretical investigation and numerical analysis have been carried out to explain the existence of the turning point in the resonance wavelength variation with the RI change. According to the basic theory, the SPR effect in the interface occurs as the light wave vector matches the SPW vector at the plane of $z=0$. The relationship between the real part of effective RI ( $\left.n_{\text {eff }}\right)$ of the core-guided mode and wavelength with the RI in the range of 1.31 to 1.35 is shown in Fig. 7, where it can be seen that the effective RI decreases with an increase in wavelength. The effective RI increases with an increase in analyte RI from 1.31 to 1.33 and decreases with an increase in analyte RI from 1.33 to 1.35 when the wavelength is in the range of 
$450 \mathrm{~nm}$ to $480 \mathrm{~nm}$. Due to the small change in analyte RI, the real part of the effective RI of core-guided mode changes, which causes the change in the phase matching wavelength between the core guided mode and the SPW mode. An increase in the effective RI of the core indicates that less light is coupled to the air layer and the metal interface, which makes the resonant wavelength shift to the shorter wavelength. The reduction of the effective $\mathrm{RI}$ of the core-guided mode indicates that more light is coupled to the air layer and the metal interface, which makes the resonant wavelength shift to the longer wavelength. Thus, an abrupt change appears in the effective RI and results in a turning point in the resonant wavelength variation with analyte RI. The electric field intensity distributions with the RI values of 1.31, 1.33, and 1.35 are shown in Fig. 8, the red checkmark represents the wavelength in which the electric field intensity of the core reaches

the minimum value, and the strongest resonance effect is achieved. It can be seen from Fig. 8 that the resonance wavelengths at the RI values of $1.31,1.33$, and 1.35 are $470 \mathrm{~nm}, 460 \mathrm{~nm}$, and $465 \mathrm{~nm}$, respectively, and there is a turning point of the resonance wavelength at the RI value of 1.33 .

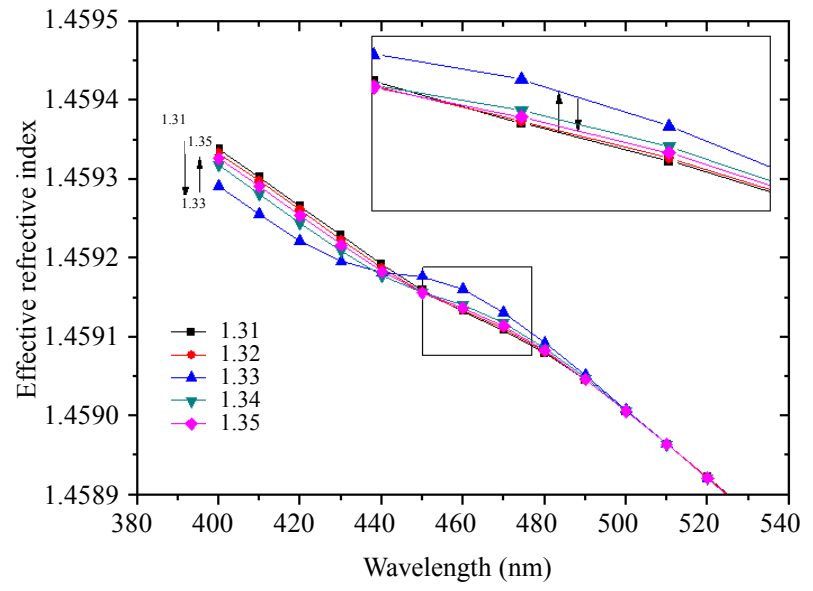

Fig. 7 Effective RI with analyte RI variation from 1.31 to 1.35 .
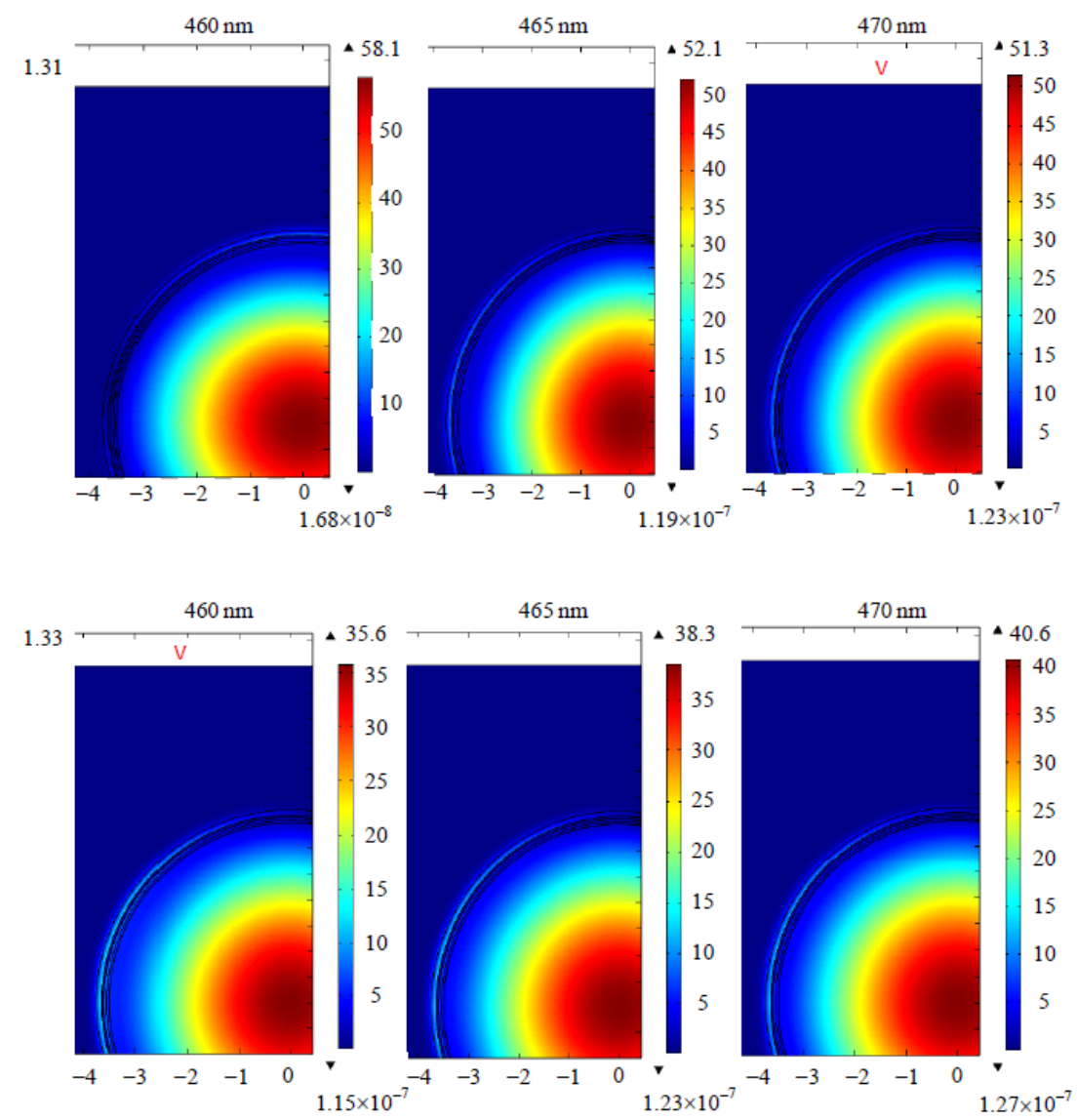


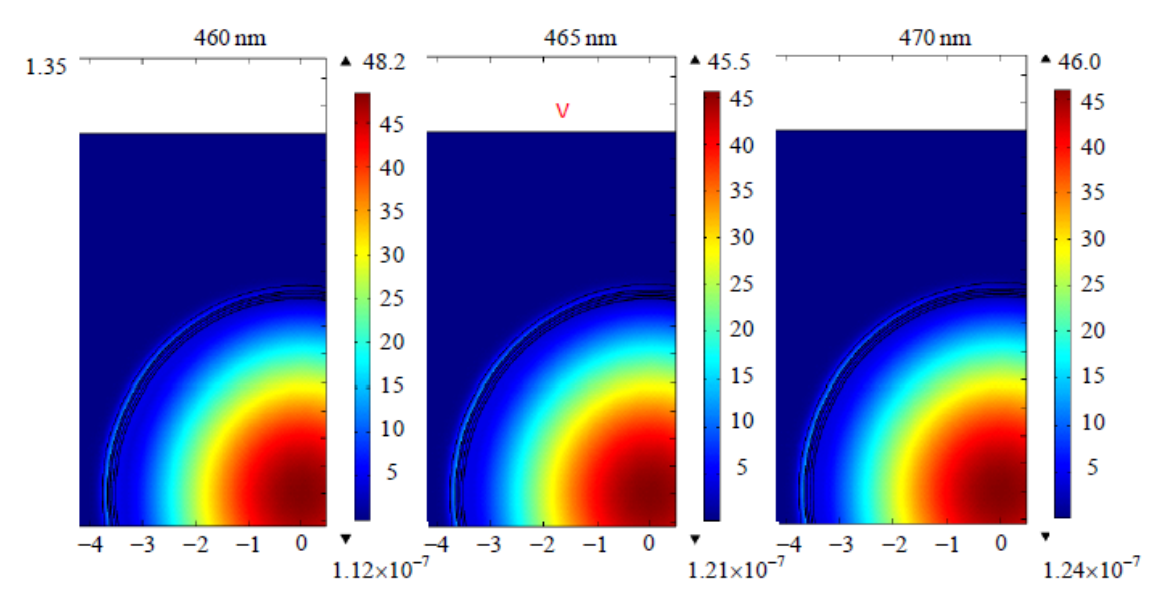

Fig. 8 Electric field intensity distributions with the refractive index are 1.31, 1.33, and 1.35.

\section{Conclusions}

In this paper, we propose a reflective type SPR RI sensor based on optical fiber microring. The sensing performance and the effects of the geometrical parameters on the resonant spectrum are investigated by use of the FEM method. Simulation results show that the monitoring range of analyte RI is from 1.0 to 1.43 . The resonance spectrum shifts toward the shorter wavelength gradually with an increase in analyte RI from 1.0 to 1.33 and shifts toward the longer wavelength gradually with an increase in analyte RI from 1.33 to 1.43 , and a turning point in the resonance wavelength at the RI value of 1.33 is observed. The highest sensitivity can be up to $2.30 \times 10^{3} \mathrm{~nm} / \mathrm{RIU}$ near the RI value of 1.0. The sensor has a large measurement range, compact size, and durable structure, and it can be used in gaseous material detections.

\section{Acknowledgment}

This work was supported by the Natural Science Foundation of Zhejiang Province China under Grant No.LY17F050010.

Open Access This article is distributed under the terms of the Creative Commons Attribution 4.0 International License (http://creativecommons.org/licenses/by/4.0/), which permits unrestricted use, distribution, and reproduction in any medium, provided you give appropriate credit to the original author(s) and the source, provide a link to the Creative Commons license, and indicate if changes were made.

\section{References}

[1] D. R. Tilley, "Surface polaritons: electromagnetic waves at surfaces and interfaces," Journal of Modern Optics, 1983, 30(11): 1501-1506.

[2] J. Homola, S. S. Yee, and G. Gauglitz, "Surface plasmon resonance sensors: review," Sensors and Actuators B: Chemical, 1999, 54(1): 3-15.

[3] Y. Wang, S. Meng, Y. Liang, L. Li, and W. Peng, "Fiber-optic surface plasmon resonance sensor with multi-alternating metal layers for biological measurement," Photonic Sensors, 2013, 3(3): 202-207.

[4] C. Perrotton, R. J. Westerwaal, N. Javahiraly, M. Slaman, H. Schreuders, B. Dam, et al., "A reliable, sensitive and fast optical fiber hydrogen sensor based on surface plasmon resonance," Optics Express, 2013, 21(1): 382-390.

[5] S. K. Srivastava, R. Verma, and B. D. Gupta, "Surface plasmon resonance based fiber optic sensor for the detection of low water content in ethanol," Sensors and Actuators B: Chemical, 2011, 153(1): 194-198.

[6] A. Nooke, U. Beck, A. Hertwig, A. Krause, H. Krüger, V. Lohse, et al., "On the application of gold based SPR sensors for the detection of hazardous gases," Sensors and Actuators B: Chemical, 2010, 149(1): 194-198.

[7] M. Xue, Q. Jiang, C Zhang, and J. Lin, "A kind of biomolecular probe sensor based on TFBG surface plasma resonance," Photonic Sensors, 2015, 5(2): 102-108.

[8] J. Homola, J. Dostalek, S. Chen, A. Rasooly, S. Jiang, and S. S. Yee, "Spectral surface plasmon resonance biosensor for detection of staphylococcal enterotoxin $\mathrm{B}$ in milk," International Journal of Food Microbiology, 2002, 75(1): 61-69.

[9] D. F. Santos, A. Guerreiro, and J. M. Baptista, 
"Numerical investigation of a refractive index SPR D-type optical fiber sensor using COMSOL Multiphysics," Photonic Sensors, 2013, 3(1): 61-66.

[10] H. Y. Lin, C. H. Huang, G. L. Cheng, N. K. Chen, and H. C. Chui, "Tapered optical fiber sensor based on localized surface plasmon resonance," Optics Express, 2012, 20(19): 21693-21701.

[11] L. C. C. Coelho, J. M. M. M. D Almeida, H. Moayyed, J. L. Santos, and D. Viegas, "Multiplexing of surface plasmon resonance sensing devices on etched single-mode fiber," Journal of Lightwave Technology, 2015, 33(2): 432-438.

[12] J. Zhu, L Qin, S. Song, J. Zhong, and S. Lin, "Design of a surface plasmon resonance sensor based on grating connection," Photonic Sensors, 2015, 5(2): 159-165.

[13] R. Verma, A. Sharma, and B. Gupta, "Modeling of tapered fiber-optic surface plasmon resonance sensor with enhanced sensitivity," IEEE Photonics Technology Letters, 2007, 19(22): 1786-1788.

[14] A. Jian, L. Den, S. Sang, Q. Duan, X. Zhang, and W. Zhang, "Surface plasmon resonance sensor based on an angled optical fiber," IEEE Sensors Journal, 2014, 14(9): 3229-3235.

[15] A. Hassani and M. Skorobogatiy, "Design of the microstructured optical fiber-based surface plasmon resonance sensors with enhanced microfluidics," Optics Express, 2006, 14(24): 11616-11621.

[16] M. Hautakorpi, M. Mattinen, and H. Ludvigsen, "Surface-plasmon-resonance sensor based on three-hole microstructured optical fiber," Optics Express, 2008, 16(12): 8427-8432.

[17] M. Erdmanis, D. Viegas, M. Hautakorpi, S. Novotny, J. L. Santos, and H. Ludvigsen, "Comprehensive numerical analysis of a surface-plasmon-resonance sensor based on an H-shaped optical fiber," Optics
Express, 2011, 19(15): 13980-13988.

[18] X. Yu, Y. Zhang, S. Pan, P. Shum, M. Yan, Y. Leviatan, et al., "A selectively coated photonic crystal fiber based surface plasmon resonance sensor," Journal of Optics, 2009, 12(1): 74-77.

[19] Y. Lu, C. J. Hao, B. Q. Wu, M. Musideke, L. C. Duan, W. Q. Wen, et al., "Surface plasmon resonance sensor based on polymer photonic crystal fibers with metal nanolayers," Sensors, 2013, 13(1): 956-965.

[20] A. K. Mishra, S. K. Mishra, and B. D. Gupta, "Gas-clad two-way fiber optic SPR sensor: a novel approach for refractive index sensing," Plasmonics, 2015, 10(5): 1071-1076.

[21] P. J. A. Sazio, A. Amezcua-Correa, C. E. Finlayson, J. R. Hayes, T. J. Scheidemantel, N. F. Baril, et al., "Microstructured optical fibers as high-pressure microfluidic reactors," Science, 2006, 311(5767): 1583-1586.

[22] J. A. Harrington, "A review of IR transmitting, hollow waveguides," Fiber \& Integrated Optics, 2000, 19(3): 211-227.

[23] N. Takeyasu, T. Tanaka, and S. Kawata, "Metal deposition deep into microstructure by electroless plating," Japanese Journal of Applied Physics, 2005, 44(35): L1134-L1137.

[24] Y. Zhu, M. Cheng, H. Wang, Y. Zhang, and J. Yang, "Design of a surface-plasmon-resonance sensor based on a microstructured optical fiber with annular-shaped holes," Plasma Science and Technology, 2014, 16(9): 867-872.

[25] A. Hassani and M. Skorobogatiy, "Design criteria for microstructured-optical-fiber based surface plasmon resonance sensors," JOSA B, 2007, 24(6): 1423-1429. 